\title{
Dielectrics for output windows of medium power gyrotrons
}

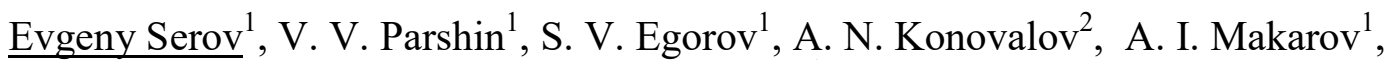 \\ K. V. Vlasova ${ }^{1}$ \\ ${ }^{1}$ Institute of Applied Physics of the Russian Academy of Sciences, Nizhny Novgorod, Russia, serov@ipfran.ru \\ ${ }^{2}$ Quartz technologies Ltd, Shilovo, Ryazan' region, Russia
}

For achieving optimal characteristics of gyrotron it is necessary to choose a suitable material for output window. Balance of relatively low dielectric losses, slowly dependent on temperature, and relatively high thermal conductivity are required for good heat removal. Certainly, modern low loss CVD diamond windows meet these requirements, but their price is too expensive for wide application.

In this paper we present an analysis of properties of several dielectric materials: boron nitride $(\mathrm{BN})$, which is currently widely used for gyrotron output windows production, crystal Quartz, and Spinel.

Basing on experimentally measured dielectric losses of these materials and on their thermal properties we made a calculation of maximal achievable output power that can be transmitted through disks made of such materials.

\section{Boron nitride}

Most of currently produced medium power $(\sim 10-100 \mathrm{~kW})$ gyrotrons have output windows made of hexagonal pyrolytic BN. Its dielectric properties in sub- $\mathrm{THz}$ range were studied in our previous works $[1,2]$. Usual samples of $\mathrm{BN}$ have $\tan \delta \approx 10^{-3}$ at $140 \mathrm{GHz}$ which is higher in comparison with other investigated materials. But the loss tangent temperature dependence is rather weak: it increases by only about $40 \%$ with temperature increasing from 25 to $700^{\circ} \mathrm{C}$.

Another important characteristic is thermal conductivity of BN. Usual value used as estimate in practice is $45 \mathrm{~W} / \mathrm{m} \cdot \mathrm{K}$ for the direction parallel the layers. However, according to the available publications $[3,4]$, thermal conductivity of $\mathrm{BN}$ can reach at least $220 \mathrm{~W} / \mathrm{m} \cdot \mathrm{K}$ for samples with high crystal perfection.

\section{Spinel}

Spinel $\left(\mathrm{MgAl}_{2} \mathrm{O}_{4}\right)$ is ceramic material with relatively low dielectric loss which is resistant to high temperatures. In our work we studied spinel samples obtained by microwave heating of ultrafine powder components [5].

Measurements of dielectric parameters ( $\mathrm{n}$ and $\tan \delta$ ) were made using high-Q resonator technique (see [1] and Refs. therein) in the frequency range $50-300 \mathrm{GHz}$ at temperatures $20-200^{\circ} \mathrm{C}$. Unlike BN, spinel demonstrates rather strong temperature dependence of dielectric loss. It is illustrated in Fig. 1. At temperature of about $400^{\circ} \mathrm{C}$ dielectric losses in spinel become higher than that in $\mathrm{BN}$.

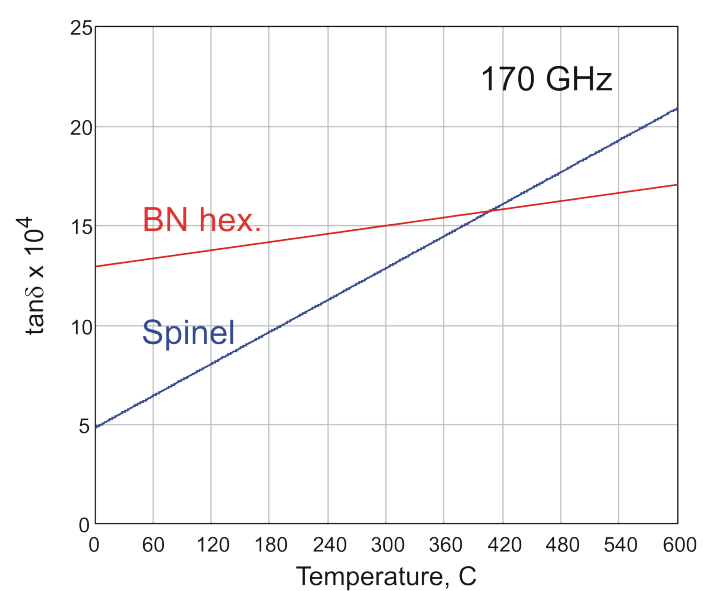

Fig. 1. Linear approximations of $\tan \delta$ temperature dependencies at $\mathrm{f}=170 \mathrm{GHz}$ based on experimental data for spinel and hexagonal $\mathrm{BN}$.

Thermal properties of spinel were taken from [6]. In comparison with $\mathrm{BN}$ it has several times lower thermal conductivity $(18 \mathrm{~W} / \mathrm{m} \cdot \mathrm{K}$ at room temperature).

\section{Quartz}

Crystal quartz produced by Quartz Technologies Ltd was grown by the method of hydrothermal synthesis on the own raw material base of the deposit "Zhelannoe". The total concentration of impurities including alkali, gas-liquid and mineral elements does not exceed 2-3 ppm (chemical composition studies were carried out at the G.G. Devyatykh Institute of High-Purity Chemistry, ANZAPLAN analytical laboratories and QSIL laboratories). It has extremely low absorption coefficient in the optical range $\left(\leq 10^{-6} \mathrm{~cm}^{-1}\right.$ at $\lambda=1 \mu \mathrm{m})$.

Investigation of quartz sample dielectric properties was made in frequency range $65-200 \mathrm{GHz}$ at room temperature. Due to specific geometric sizes of the sample only $\mathrm{n}_{\mathrm{o}}$ component and corresponding value of $\tan \delta$ were measured. Fig. 2 demonstrates experimentally measured frequency dependence of dielectric losses in quartz along the main axis. The losses in this sample are practically the lowest ones in comparison with earlier measured samples [1].

Thermal conductivity in quartz was taken from paper [7]: $6 \mathrm{~W} / \mathrm{m} \cdot \mathrm{K}$ for [010] direction and $13 \mathrm{~W} / \mathrm{m} \cdot \mathrm{K}$ for [001] direction at room temperature. 


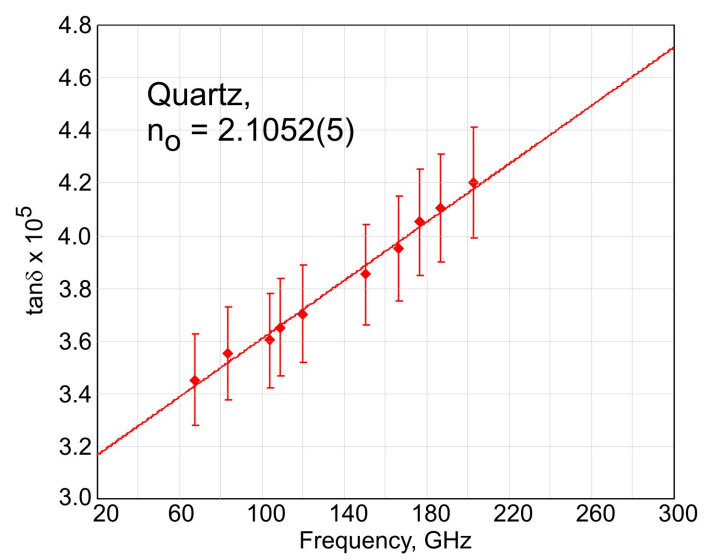

Fig. 2 Experimentally measured frequency dependence of dielectric losses in quartz for the direction parallel to the main crystal axis. Line is the linear regression.

\section{Thermal calculation and discussion}

Using data on dielectric losses and thermal properties of the samples we made a calculation of temperature distribution for a disks used as gyrotron output windows in the continuous-wave operation (CW). The calculation was made in a similar way as in [8]. We used Newton's law of cooling as the boundary condition on the perimeter. The coolant temperature was $30^{\circ} \mathrm{C}$, and the coefficient of heat removal from the window perimeter was $4 \mathrm{~W} / \mathrm{cm}^{2} \cdot \mathrm{K}$. Heat flows from the window-vacuum and window-air boundaries were neglected.

The maximal output power for gyrotron windows made of $\mathrm{BN}$ and spinel was found as maximal beam power for which the temperature of the disk did not exceed $600^{\circ} \mathrm{C}$. The thermal conductivity of $\mathrm{BN}$ was taken as $45 \mathrm{~W} / \mathrm{m} \cdot \mathrm{K}$. For quartz window the calculation is more estimative because we do not have information about the temperature dependence of $\tan \delta$. Besides, it is unlikely that quartz disk will withstand a large temperature gradient arising when the temperature of the disk center rises to $600^{\circ} \mathrm{C}$. Thus, in our estimation of maximal power we limited maximal temperature of quartz to $300^{\circ} \mathrm{C}$. For more strict calculation additional factors should be taken into account.

The window thickness was resonant, i.e., the optical thickness was equal to an integer number of halfwavelengths. The number of the half-wavelengths was chosen for the reason of the window strength. For disks with diameters of about $100 \mathrm{~mm}$, the thicknesses ranged from 3 to $4 \mathrm{~mm}$, and for disks with diameters of $30 \mathrm{~mm}$ (for $\mathrm{f} \sim 30 \mathrm{GHz}$ ), the thicknesses was 2-2.5 mm. Fig. 3 shows the maximal power transmitted through the window.

On the basis of Fig. 3 we can conclude that $\mathrm{MgAl}_{2} \mathrm{O}_{4}$ spinel is not optimal material for gyrotron output windows. Crystal quartz is a more suitable one, comparable to boron nitride in terms of maximum power level and even surpassing it at high frequencies. However, some problems associated with the use of crystal quartz may occur due to difficulty of soldering and possible mechanical stresses. Additional data on temperature dependence of $\tan \delta$ are required.

Comparison of Fig. 3 (above) and Fig. 5 from [8] leads us to the conclusion that $6 \mathrm{H}-\mathrm{SiC}$ disk can transmit several times more powerful beam than $\mathrm{BN}$ or quartz disks. But if we use BN with high crystal perfection and high thermal conductivity then such material can also provide several hundred kilowatts level for the $\mathrm{CW}$ mode in the sub-THz range.

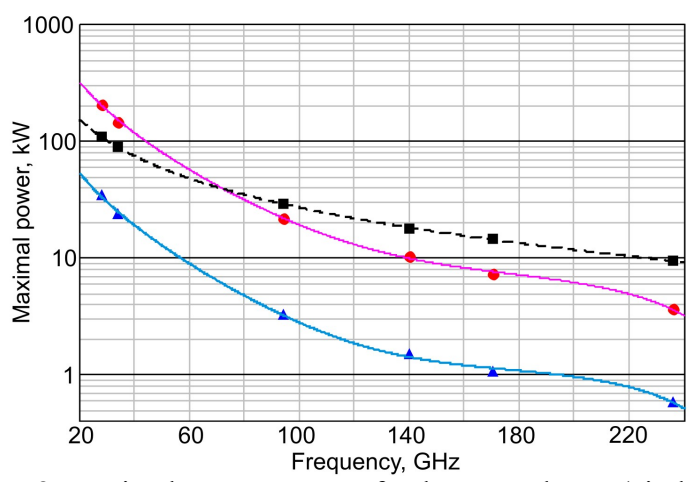

Fig. 3 Maximal output power for hexagonal BN (circles), spinel (triangles) and quartz (squares). Curves are smooth approximations. See text for details.

\section{Acknowledgements}

The work was supported by the Russian Foundation for Basic Research and the Government of the Nizhny Novgorod region, project No. 18-42-520015.

\section{References}

1. Parshin, V. V., Tretyakov, M. Yu., Koshelev, M. A., Serov, E.A. Instrumental complex and the results of precise measurements of millimeter- and submillimeter-wave propagation in condensed media and the atmosphere // Radiophys. \& Quant. Electron. 2009, V. 52, No. 8. P. 525535 .

2. Parshin, $V$. V. Dielectric materials for gyrotron output windows // International Journal of Infrared and Millimeter Waves. 1994, V. 15, No. 2. P. 339-348.

3. Sichel, E. K., Miller, R. E., Abrahams, M. S., Buioc$c h i, C$. J. Heat capacity and thermal conductivity of hexagonal pyrolytic boron nitride // Phys. Rev. B. 1976, V. 13, No. 10. P. 4607-4611.

4. Duclaux, L., Nysten, B., Issi, J-P. Structure and lowtemperature thermal conductivity of pyrolytic boron nitride // Phys. Rev. B. 1992, V. 46, No. 6. P. 3362-3367.

5. Egorov, S. V., Bykov, Yu. V., Eremeev, A. G., Sorokin, A. A., Serov, E.A., Parshin, V. V., Balabanov, S. S., Belyaev, A. V., Novikova, A. V., Permin, D. A. MillimeterWavelength Radiation Used to Sinter Radiotransparent $\mathrm{MgAl}_{2} \mathrm{O}_{4}$ Ceramics // Radiophys. \& Quant. Electron. 2017, V. 59, No. 8-9. P. 690-697.

6. Harris, D. C., Linda F. Johnson, L. F., Seaver, R., Lewis, T. Turri, G., Bass, M., Zelmon, D. E., Haynes, D. N. Optical and thermal properties of spinel with revised (increased) absorption at 4 to $5 \mu \mathrm{m}$ wavelengths and comparison with sapphire // Optical Engineering. 2013, V. 52, No.8. 087113.

7. Kanamori, H., Fujii, N., Mizutani, H. Thermal Diffusivity Measurement of Rock-Forming Minerals from $300^{\circ}$ to $1100^{\circ} \mathrm{K} / /$ Journal of Geophysical Research. 1968, V. 73, No. 2. P. 595-605.

8. Parshin, V., Serov, E., Denisov, G., Garin, B., Denisyuk, R., V'yuginov, V., Klevtsov, V., Travin, $N$. Silicon carbide for high-power applications at $\mathrm{MM}$ and $\mathrm{THz}$ ranges // Diamond \& Related Materials. 2017, V. 80, P. 1-4. 